\title{
Linear Stability Implications of Mean Flow Variations in Turbulent Jets Issuing from Serrated Nozzles
}

\author{
Aniruddha Sinha ${ }^{1 *}$ and Tim Colonius ${ }^{2 \dagger}$ \\ ${ }^{1}$ Indian Institute of Technology Bombay, Powai, Mumbai 400076, INDIA \\ ${ }^{2}$ California Institute of Technology, Pasadena, CA 11925, USA
}

\begin{abstract}
Nozzle serrations or chevrons are being deployed for reducing the noise from jet engines. The turbulent mean flow field of such jets takes on a serrated character, and the linear stability eigenspectrum for such serrated mean flows determines the evolution of the coherent wavepackets that are linked to the aft angle noise radiated. In particular, the lower the growth rate and phase speed of the instability, the lower is the expected noise radiation. Here we identify four parameters of the mean flow serrations - viz. number of lobes, their protrusion, their width relative of overall circumference, and the average thickness of the shear layer. These four parameters are systematically varied to synthesize a family of mean flow profiles. The corresponding stability analyses indicate the following trends. As expected from results for round jets, the average shear layer thickness has an inverse effect on both growth rate and phase speed. Deeper penetration and higher number of lobes reduce the growth rate of the relevant instability while mildly enhancing its phase speed. The relative width of the lobes do not appear to be a relevant parameter. These theoretical trends are supported by noise measurements in the parametric study on chevron nozzles performed at NASA.
\end{abstract}

\section{Introduction}

Jet noise is a concern for the continued expansion of jet aviation, and several passive and active control techniques are being researched to address the issue. One such solution that has been actually deployed on production aircraft is the addition of serrations at the nozzle trailing edge, which are called chevrons (see fig. 1(a)). The chevron tips impinge on the jet shear layer and generate streamwise vortices that enhance mixing and shorten the potential core. ${ }^{1,2}$ They also reduce the low frequency mixing noise at aft angles (which is the loudest component of jet noise), but typically increase high frequency noise at all angles. ${ }^{1}$ The latter phenomenon is due to enhancement of small scale turbulence near the nozzle by the impinging chevrons. $^{2}$ The low frequency reduction may be related to alterations in large scale structure dynamics, which is not understood fully as yet.

The low frequency aft angle mixing noise in round jets has been linked with large scale structure dynamics, which in turn have been successfully modeled using the linear instability modes of the turbulent mean flow field. ${ }^{3}$ This idea had been explored earlier, ${ }^{4,5,6,7}$ but the recent availability of detailed experimental data and well-validated large-eddy simulation (LES) data have given strong credence to the linear stability model. $8,9,10,11$ The models have been constructed both from the classical parallel-flow linear stability theory (hereafter simply termed LST) as well as the parabolized stability equation (PSE) that accounts for mild non-parallel effects.

The presence of nozzle serrations creates corresponding serrations in the mean flow field of the jet; hence these will be hereafter referred to as 'serrated jets'. Based on this previous modeling success in round jets, we adopt the hypothesis that the low frequency aft angle noise reduction observed in serrated jets is explained by the modification of their linear instability modes. Gudmundsson and Colonius ${ }^{12}$ lent support to this hypothesis by demonstrating qualitative similarity of the instability wave amplitude and phase evolution

\footnotetext{
*Assistant Professor, Department of Aerospace Engineering; AIAA Member; Corresponding author: as@aero.iitb.ac.in

$\dagger$ Professor, Department of Mechanical Engineering; AIAA Associate Fellow
} 


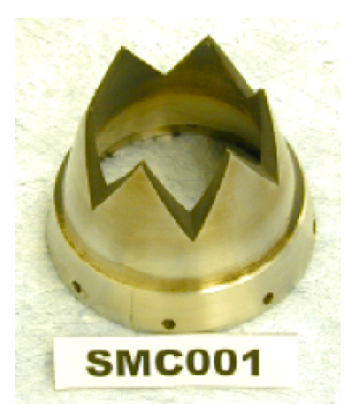

(a)

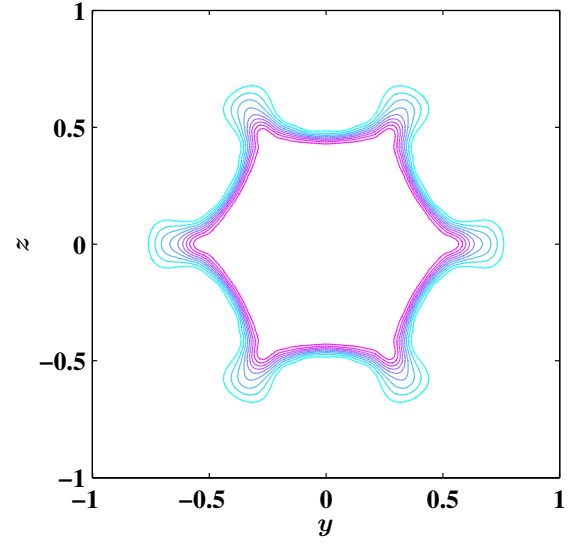

(b)

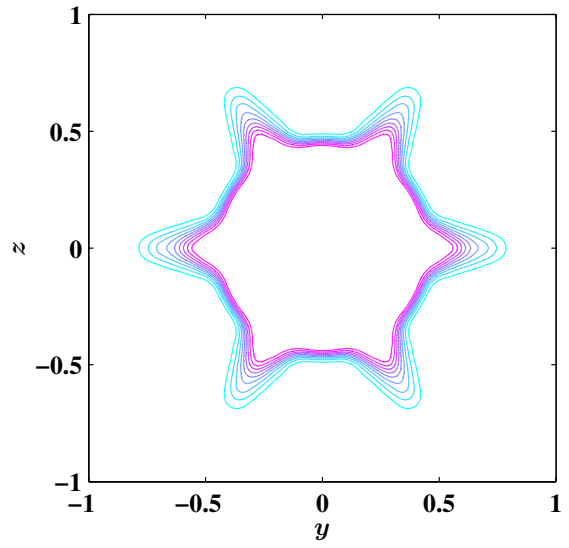

(c)

Figure 1. (a) Serrated SMC001 nozzle tested at NASA SHJAR. (b) Mean axial velocity field at $x=0.5$ in the cold Mach 0.9 jet issuing from the SMC001 nozzle. (c) The same field using an approximate parameterization. In the two latter plots, contour levels are equally spaced between 0.1 and $0.9 U_{j}$.

with empirical near-field pressure measurements. The latter were made at NASA SHJAR on a Mach 0.9 cold jet issuing from the serrated nozzle pictured in fig. 1(a).

At present, the design and development of serrated nozzles is based on empirical laboratory and full-scale testing. ${ }^{1,13}$ In this paper, we present the linear stability implications of modifications of various aspects of the serrated mean flow field at a given axial station. This is intended as a first step towards prediction of the noise impact of various of chevron nozzle designs.

The serrated mean flow shown in fig. 1(b) has lobes corresponding to the ejection of high-speed core air at the roots of the chevrons. The chevron tips impinge on the shear layer, thus creating the flats in between lobes. In this work, we modify the protrusion of the lobes, their widths (as a fraction of their azimuthal spacing), their number (corresponding to more or fewer chevrons on the nozzle), and the average thickness of the shear layer. These four aspects are systematically varied to study their effect on the growth rate and phase speed of the unstable LST eigenmodes. The latter two aspects are related to the jet mixing noise levels observed in the aft sector. ${ }^{3}$

The sensitivity of linear stability waves to base flow modifications has been analyzed earlier to investigate linear-to-turbulent transition in wall-bounded flows. ${ }^{14,15,16} \mathrm{~A}$ similar perspective was adopted to study the effect of the base flow changes in a jet on transformation from absolute to convective instability. ${ }^{17}$ Recently, Cavalieri and Agarwal ${ }^{18}$ considered the sensitivity of linear stability modes of a turbulent mixing layer with modifications of the mean flow with a view to elucidate their aeroacoustic implications. The perspective adopted herein is closest to this last work, although the method used is different.

\section{Spatial Linear Stability Theory for Jets Issuing from Serrated Nozzles}

We formulate the parallel-flow spatial linear stability theory for viscous compressible jets with a serrated mean flow field. The usual compressible formulation is used to nondimensionalize flow quantities. Linear dimensions are normalized by the nozzle exit diameter $D$, velocities by the ambient speed of sound $c_{\infty}$, density by the ambient density $\rho_{\infty}$, and pressure by $\rho_{\infty} c_{\infty}^{2}$. Time is normalized by $D / c_{\infty}$. However, for the purposes of reporting, frequency is normalized by $U_{j} / D$ to the more common Strouhal number $S t$, where $U_{j}$ is the nozzle exit velocity. The acoustic Mach number of the jet is $M_{a}=U_{j} / c_{\infty}$, whereas the jet exit Mach number is $M_{j}=U_{j} / c_{j}$, with $c_{j}$ being the speed of sound at the nozzle exit. The jet exit Reynolds number is $R e_{j}=\rho_{j} U_{j} D / \mu_{j}$, with $\rho_{j}$ and $\mu_{j}$ being respectively the density and viscosity at the nozzle exit. In the stability analysis, the temperature dependence of viscosity is ignored since the temperature ratio of the jets considered is close to unity. Moreover, the Prandtl number $\operatorname{Pr}$ is fixed at 0.7 for air.

The jet flow field is described in cylindrical coordinates $(x, r, \theta)$ by $\boldsymbol{q}=\left(u_{x}, u_{r}, u_{\theta}, p, \zeta\right)^{\mathrm{T}}$, which respectively denote the axial, radial and azimuthal components of velocity, pressure and specific volume. The 
instantaneous flow field is decomposed as fluctuations $\boldsymbol{q}^{\prime}$ of the time-averaged turbulent flow field $\overline{\boldsymbol{q}}$, i.e. $\boldsymbol{q}(x, r, \theta, t)=\overline{\boldsymbol{q}}(r, \theta)+\boldsymbol{q}^{\prime}(x, r, \theta, t)$. The streamlines of the mean flow are assumed to be locally parallel to the streamwise direction; this also implies that the mean radial and azimuthal velocities vanish. Moreover, the mean pressure is constant in the free jets under consideration, and its non-dimensional value is $1 / \gamma, \gamma$ being the ratio of the specific heats (assumed 1.4).

When the governing equations for the flow (compressible and viscous) are linearized about the mean flow field, the coefficients of the resulting PDE (in $\boldsymbol{q}^{\prime}$ and its derivatives) do not depend on time or the axial coordinate. Hence, the solution is separable in these directions and is of the form

$$
\boldsymbol{q}^{\prime}(x, r, \theta, t)=\tilde{\boldsymbol{q}}(r, \theta) \exp \{\mathrm{i}(\alpha x-\omega t)\}+\text { c.c. }
$$

Using this ansatz, the following linear governing equation is obtained in matrix form

$$
\begin{gathered}
{\left[-\mathrm{i} \omega+\left\{\overline{\mathcal{L}}^{0}+\overline{\mathcal{L}}^{r} \frac{\partial}{\partial r}+\overline{\mathcal{L}}^{r r} \frac{\partial^{2}}{\partial r^{2}}+\left(\overline{\mathcal{L}}^{\theta}+\overline{\mathcal{L}}^{r \theta} \frac{\partial}{\partial r}\right) \frac{\partial}{\partial \theta}+\overline{\mathcal{L}}^{\theta \theta} \frac{\partial^{2}}{\partial \theta^{2}}\right\}\right.} \\
\left.+\mathrm{i} \alpha\left\{\overline{\mathcal{L}}^{x}+\overline{\mathcal{L}}^{x r} \frac{\partial}{\partial r}+\overline{\mathcal{L}}^{\theta x} \frac{\partial}{\partial \theta}\right\}-\alpha^{2} \overline{\mathcal{L}}^{x x}\right] \tilde{\boldsymbol{q}}(r, \theta)=\mathbf{0} .
\end{gathered}
$$

The $5 \times 5$ matrices $\overline{\mathcal{L}}$ are linear functions of $\overline{\boldsymbol{q}}$ in general, and are parametrized by $\operatorname{Re}_{j}, M_{a}$ and $\operatorname{Pr}$.

For the convectively unstable flows under consideration, it is appropriate to study the spatial growth/decay and phase speed of disturbances at specified real frequencies - the spatial analysis. ${ }^{19}$ In this case, $\omega$ $\left(=2 \pi S t M_{a}\right)$ is a given real quantity and the governing equations determine the complex $\alpha$ as an eigenvalue with the real part $\alpha^{r}$ and imaginary part $\alpha^{i}$ respectively implying the axial wavenumber and negative growth (decay) rate. The mode shape $\tilde{\boldsymbol{q}}$ is the associated eigenvector. Thus, eqn. (2) is a 2-D $(r, \theta)$ quadratic eignevalue problem.

We neglect the $\alpha^{2}$ term in eqn. (2) (arising due to viscosity) to obtain the usual linear eigenvalue problem with half the dimensionality. Due to the absence of solid boundaries and the high Reynolds number of the flows considered here $\left(\sim 10^{6}\right)$, this term is expected to have a minor effect on the stability results. ${ }^{20,21}$ The other viscous terms are retained to avoid any special treatment of the critical layer. ${ }^{22}$

Equation (2) can be solved directly with intensive computation. However, the symmetries of the corrugated mean flow can be exploited to significantly reduce the problem size while developing further insight into the instability characteristics. We start by defining the $m$ th azimuthal Fourier mode of, say $T(\theta)$, as $\hat{T}_{m}:=(1 / 2 \pi) \int_{-\pi}^{\pi} T(\theta) \mathrm{e}^{-\mathrm{i} m \theta} \mathrm{d} \theta$. Our nozzle has $L$ chevrons distributed uniformly around the circumference $(L=6$ in fig. 1(a)). Jet flow control actuators are typically distributed uniformly in $\theta$ also. In either case, the resulting turbulent mean flow field has an $L$-fold rotational symmetry so that $\hat{\overline{\boldsymbol{q}}}_{m}$ vanishes for all $m$ that are not integer multiples of $L$. That is,

$$
\overline{\boldsymbol{q}}(r, \theta)=\sum_{j=-\infty}^{\infty} \hat{\overline{\boldsymbol{q}}}_{L j}(r) \mathrm{e}^{\mathrm{i} L j \theta} .
$$

Since the operators $\overline{\mathcal{L}}$ are linear functions of $\overline{\boldsymbol{q}}$, they can be expanded in the above fashion also. Performing an azimuthal Fourier transform of eqn. (2), and substituting such expansions of $\overline{\mathcal{L}}$ in it, we obtain the following coupled set of 1-D eigenvalue problems

$$
\begin{array}{r}
-\mathrm{i} \omega \hat{\tilde{\boldsymbol{q}}}_{m}+\sum_{j=-\infty}^{\infty}\left[\hat{\overline{\mathcal{L}}}_{L j}^{0}+\hat{\overline{\mathcal{L}}}_{L j}^{r} \frac{\partial}{\partial r}+\hat{\overline{\mathcal{L}}}_{L j}^{r r} \frac{\partial^{2}}{\partial r^{2}}+\mathrm{i}(m-L j)\left(\hat{\overline{\mathcal{L}}}_{L j}^{\theta}+\hat{\overline{\mathcal{L}}}_{L j}^{r \theta} \frac{\partial}{\partial r}\right)\right. \\
\left.-(m-L j)^{2} \hat{\overline{\mathcal{L}}}_{L j}^{\theta \theta}+\mathrm{i} \alpha\left\{\hat{\overline{\mathcal{L}}}_{L j}^{x}+\hat{\overline{\mathcal{L}}}_{L j}^{x r} \frac{\partial}{\partial r}+\mathrm{i}(m-L j) \hat{\overline{\mathcal{L}}}_{L j}^{\theta x}\right\}\right] \hat{\tilde{\boldsymbol{q}}}_{m-L j}=\mathbf{0} .
\end{array}
$$

This indicates that a given azimuthal mode, say $l$, is only coupled with other azimuthal modes in the set $\left\{\hat{\tilde{\boldsymbol{q}}}_{l-L k}\right\}_{k=-\infty}^{\infty}$. Evidently, there are only $L$ unique sets of this kind, each of which represents a solution of eqn. (4) associated with a particular eigenvalue. We index these sets by the lowest azimuthal mode appearing in them; i.e., $\hat{\tilde{\boldsymbol{Q}}}^{M}:=\left\{\hat{\tilde{\boldsymbol{q}}}_{M-L k}\right\}_{k=-\infty}^{\infty}$, for $-L / 2<M \leq L / 2$. We term the set $\hat{\tilde{\boldsymbol{Q}}}^{M}$ as the $M$ th 'azimuthal order' of the eigen-solution. These azimuthal orders also represent the $L$ separable solutions (now indexed by $M$ ) of the $2-\mathrm{D}$ eigenvalue problem in eqn. (2):

$$
\tilde{\boldsymbol{q}}^{M}(r, \theta):=\sum_{k=-\infty}^{\infty} \hat{\tilde{\boldsymbol{q}}}_{M-L k}(r) \mathrm{e}^{\mathrm{i}(M-L k) \theta} .
$$


The infinite sums indicated in eqns. (3-5) must be truncated in the computation. We assume that the azimuthal complexity of $\overline{\boldsymbol{q}}(r, \theta)$ is such that the summation in eqn. (3) can be truncated to $\pm J$. For the calculations presented herein, $J=3$ was found to be adequate. Since the matrices $\overline{\mathcal{L}}$ are linear functions of $\overline{\boldsymbol{q}}$, the summation in eqn. (4) is also truncated to $\pm J$. The summation in eqn. (5) (and hence the degree of azimuthal coupling in eqn. (4)) is also truncated to $\pm N(\geq J)$. The rationale is that high azimuthal modes tend to have negligible impact on the dynamics of the low azimuthal modes, which are most unstable in general. Converged solutions are found in the present calculations with $N=9$.

Since the mean flow loses its serrated character far enough away from the control devices, the individual azimuthal modes in eqn. (4) decouple at $r \rightarrow \infty$. Thus, we use characteristic boundary conditions ${ }^{23}$ to close the outer domain at $r=10$, as in a round jet. ${ }^{9}$ The centerline behavior of each azimuthal mode is formulated as a Fourier azimuthal counterpart of the pole condition proposed by Mohseni \& Colonius $^{24}$ (see also Sinha et al. $\left.{ }^{11}\right)$.

The radial grid is clustered near the lip-line using an erf mapping. ${ }^{25}$ With 800 grid points, the minimum spacing is $\Delta r=0.0032$. Fourth-order central difference is used to discretize the radial derivative operators. The resulting matrix eigenvalue problem is solved with the Arnoldi algorithm using the parallel computing version of the ARPACK software library (see http://www.caam.rice.edu/software/ARPACK/).

The individual serrations of the mean flow field are mirror-symmetric about their center planes. We choose the reference of the azimuthal coordinate system to coincide with one such plane, so that the mean axial velocity field is even in $\theta$. These symmetries of the mean flow field bestow corresponding symmetries to the eigen-solutions.

\section{Modifications of the Jet Mean Flow Profile}

One could approach the problem of ascertaining stability implications of the mean flow profile in serrated jets by interrogating RANS or experimental flow fields. However, a sufficiently diverse dataset is not available to us. Hence, we are improvising by synthesizing mean flow profiles that we believe will represent the effects of various chevron geometries.

The baseline jet of interest is the Mach 0.9 cold jet issuing from the SMC001 nozzle (see fig. 1(a)) in experiments conducted at the NASA SHJAR facility. In the parallel-flow LST, we study the stability characteristics of the mean flow field at each cross-section of the jet separately. The 'baseline mean flow field' (modifications of this are investigated later) is that measured at $x=0.5$ in the above jet; the axial velocity contours of this field are depicted in fig. 1(b). In this work, we will study the linear stability implications of four aspects of this type of mean flow field, as discussed in $\S$ I. This requires an efficient parameterization of the flow field.

Let the mean axial velocity at the centerline and that in co-flow be $U_{\mathrm{cl}}$ and $U_{\mathrm{co}}$, respectively, at the axial station of interest. Then the 'normalized mean axial velocity' is defined as

$$
\bar{u}_{x}^{n}(x, r, \theta):=\frac{\bar{u}_{x}(x, r, \theta)-U_{\mathrm{co}}(x)}{U_{\mathrm{cl}}(x)-U_{\mathrm{co}}(x)},
$$

so that the new quantity is in the range $[0,1]$.

Figure 2(a) shows five of the contours of the baseline normalized mean flow field, abstracted for the minimal unique azimuthal sector, viz. $0^{\circ}$ to $30^{\circ}$. This azimuthal span is rescaled to the range $[0,1]$, and we denote this scaled variable as $\xi$. The particular contour levels are chosen since they are approximately equally spaced in $r$.

The $\bar{u}_{x}^{n}=0.5$ contour (radial location as a function of $\xi$ ) is fitted with the following function:

$$
c_{0.5}(\xi)=b_{0.5}+0.5 h_{0.5}\left\{1-\cos \left(\pi(1-\xi)^{-1 / \log _{2}\left(1-w_{0.5}\right)}\right)\right\} .
$$

Here, $b_{0.5}$ establishes the radial location of the contour at $\xi=1, h_{0.5}$ corresponds to the additional radial protrusion of the lobe at $\xi=0$, and $w_{0.5}$ determines the lobe's half width at half maximum (HWHM). The parameters are determined by nonlinear least squares fit in MATLAB; as an example, the parameters for the present contour are $b_{0.5}=0.463, h_{0.5}=0.175$, and $w_{0.5}=0.255$. The resulting fitted contour is shown in fig. 2(a) with a dotted line, and demonstrates an acceptable three-parameter match with the original. The fitting function in eqn. (8) is designed so that the contour has zero $\xi$-slope at the tip and root of the lobes (i.e., at $\xi=0$ and 1 , respectively), as long as $w_{0.5} \in[0,1]$. 


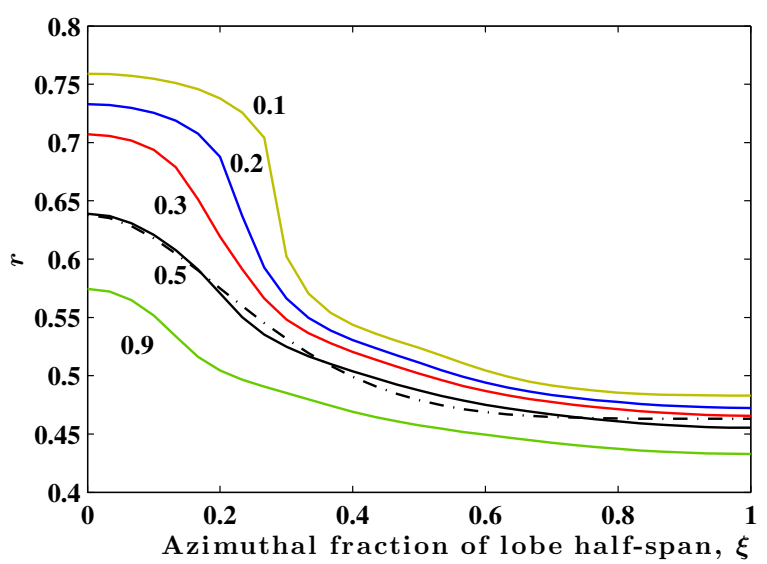

(a)

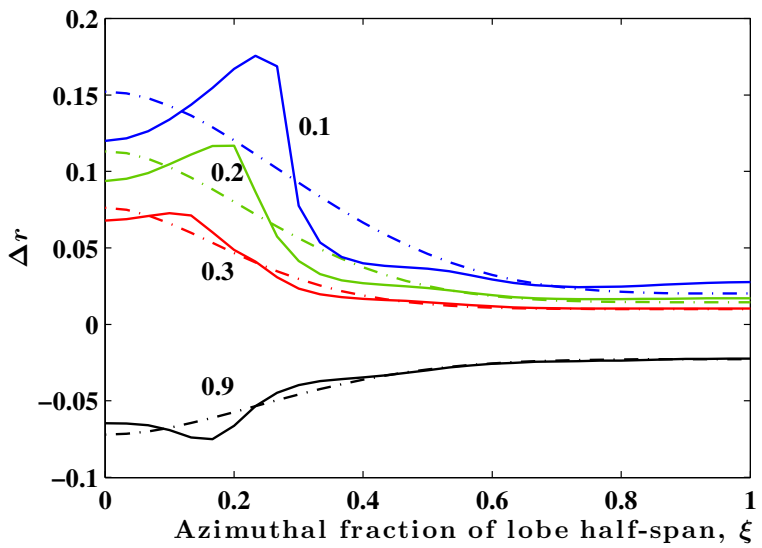

(b)

Figure 2. (a) Salient contours of normalized mean axial velocity in the baseline field presented for the minimal unique azimuthal sector, which in turn is rescaled to the range $[0,1]$. (b) The same contours depicted as departures from the 0.5-level contour. Solid curves are from the data; dotted curves are functional fits.

The $\bar{u}_{x}^{n}=0.5$ contour is supposed to be 'anchoring' the mean flow field contour plot. Hence, the remaining four contours are now presented as radial departures from this anchoring contour in fig. 2(b). Apart from an additional 'hump', the curves do not appear too dissimilar from the anchoring contour itself. Hence they are also fitted with functions of the form presented in eqn. (8). For example, the $\bar{u}_{x}^{n}=0.2$ contour is fitted with the following function:

$$
c_{0.2}(\xi)=c_{0.5}(\xi)+b_{0.2}+0.5 h_{0.2}\left\{1-\cos \left(\pi(1-\xi)^{-1 / \log _{2}\left(1-\zeta_{0.2} w_{0.5}\right)}\right)\right\} .
$$

Here, $\zeta_{0.2}$ represents a fraction of the lobe HWHM, so that subsequent modifications of the latter lead to correct behavior of the shear layer thickness (see sample mean flow fields later). The fitted contours are presented in fig. 2(b) for visual comparison.

Next we attempt to recreate the mean axial velocity field from the fifteen parameters extracted above (three for each of the five contours). At any fixed value of $\xi$, the five fitting functions are evaluated to retrieve the approximate radial locations of the respective contour levels. These are, in turn, fitted with the following function:

$$
\begin{aligned}
& V(r)=(1-\beta) \mathcal{G}\left(r ; s_{1}, q_{1}\right)+\beta \mathcal{G}\left(r ; s_{2}, q_{2}\right), \quad \beta \leq 0.5, \\
& \mathcal{G}(r ; s, q)= \begin{cases}1, & 0 \leq r<s, \\
\exp \left\{-\ln 2 \frac{(r-s)^{2}}{q^{2}}\right\}, & r \geq s .\end{cases}
\end{aligned}
$$

Here, $\mathcal{G}$ is a truncated Gaussian function of the kind used to fit mean axial velocity profiles in round jets. ${ }^{9}$ Two such functions are necessitated by the additional complexities present in the profiles in jets issuing from serrated nozzles. The five fitting parameters necessitate the identification of the five contours in the previous step.

The approximate mean axial velocity field in the $r-\xi$ domain is subsequently retrieved from this fitted normalized field by applying the inverse of the scaling described in eqn. (6). The full azimuthal variation of the field is obtained by mapping the $\xi$ domain back to the $\theta$ domain, and replicating (after mirroring) the above sectoral field suitably. The outcome of this entire fitting exercise is shown in fig. 1(c). Overall, the contours resemble those in the original field in fig. 1(b) in terms of the four main aspects laid out in $\S \mathrm{I}$.

Now that an efficient parameterization of the mean flow field is obtained, we can modify its four aspects, either in isolation or simultaneously. Variations in the protrusion and width of the lobes of the mean flow field can be achieved by suitably varying $h_{0.5}$ and $w_{0.5}$, respectively. This directly changes the anchoring $\bar{u}_{x}^{n}=0.5$ contour, and the other contours also shift with it. Fractional changes in the average shear layer thickness can be implemented by like fractional changes in all the four $b$ 's and four $h$ 's defining the secondary 

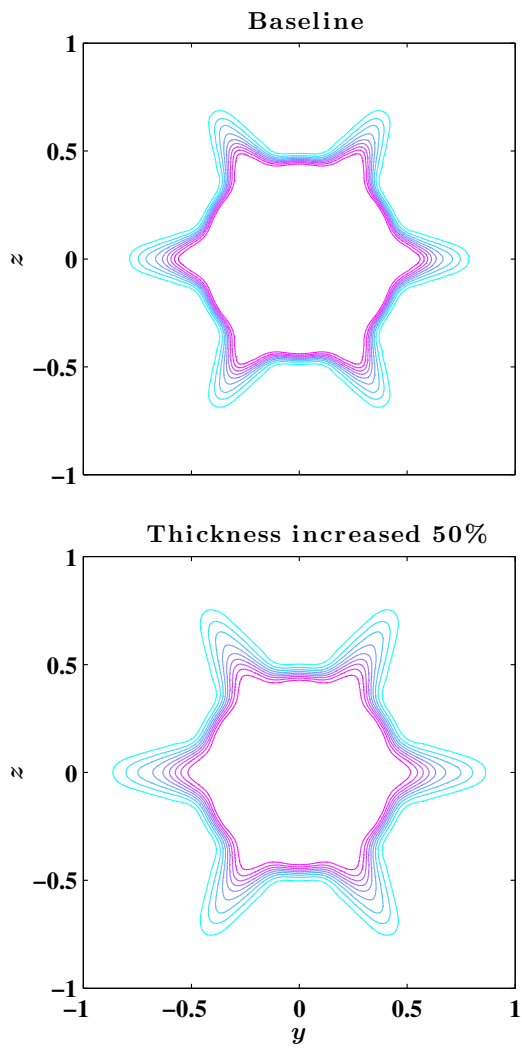

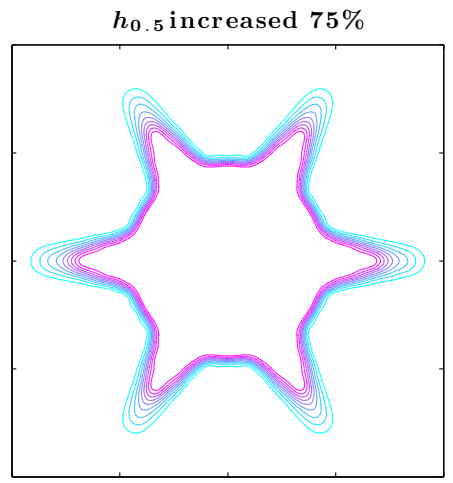

No. of lobes $=8$

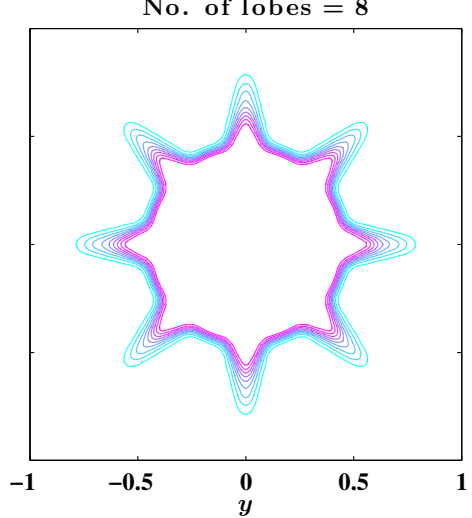

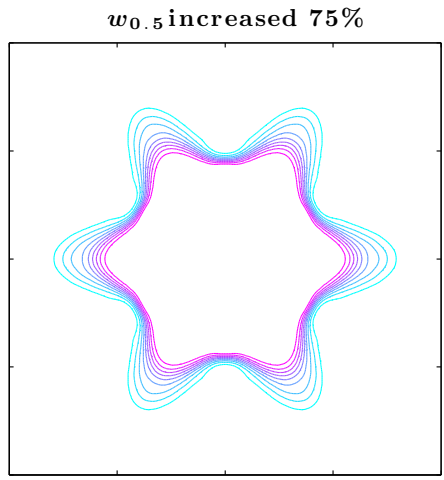

All above changes together

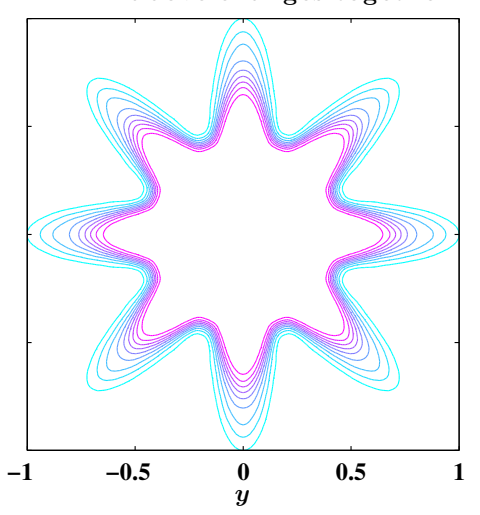

Figure 3. Sample modifications of the mean flow field contours. All modifications are reported as fractions of the baseline values, except for the number of lobes.

contour levels, leaving the anchoring contour intact. Finally, the number of lobes can be varied by changing the $\xi \rightarrow \theta$ mapping in the final step. For example, although $\xi \in[0,1]$ is mapped to $\theta \in\left[0^{\circ}, 30^{\circ}\right]$ to obtain 6 lobes, we can instead map to $\theta \in\left[0^{\circ}, 22.5^{\circ}\right]$ to have 8 lobes. Figure 3 presents some sample modifications of the baseline field, which attest to the effectiveness of the proposed parameterization.

It is difficult to directly link these aspects of the mean flow field with the geometry of the chevrons, apart from the number of lobes. It must also be remembered that these aspects of the mean flow field change as one proceeds downstream from the nozzle exit. Allowing for these caveats, we posit the following relations. Making the chevrons impinge more on the shear layer may increase the protrusions of the lobes, as they represent the ejection of high-speed air between the chevrons. If the chevrons are made more stubby (i.e., their tip angle is increased), the lobe HWHM may increase. Both these chevron modifications will also affect the shear layer thickness, and more importantly, the change of the thickness with downstream distance. Thus, the actual modification of the mean flow field can only be found through experiments or RANS/LES.

\section{Review of Stability Results for Baseline Mean Flow}

The detailed stability analysis of the Mach 0.9 cold jet issuing from the SMC001 nozzle has been presented elsewhere; ${ }^{12}$ here we only highlight the main results. Figure $4(\mathrm{a})$ is the eigenspectrum found at two axial stations near the nozzle for the $M=0,1$ and $2, S t=0.35$ mode. For comparison, the results for the corresponding round nozzle (SMC000) tested at NASA SHJAR is also presented. The serrated jet displays multiple unstable eigenmodes, as opposed to the single unstable eigenmode presented by the round jet. Furthermore, although the serrated jet is more unstable at $x=0.5$ compared to the round jet, it becomes substantially less so at $x=2.0$. It is hypothesized that the modifications in growth rates and phase speeds seen in fig. 4(a) are accountable for the reduction in low frequency mixing noise in serrated jets.

The pressure eigenfunctions corresponding to the three unstable eigenmodes in $S t=0.35, M=0$ at $x=0.5$ are shown in fig. $4(\mathrm{~b})$. Mode (i), the most unstable at this station, is seen to peak at the flats 

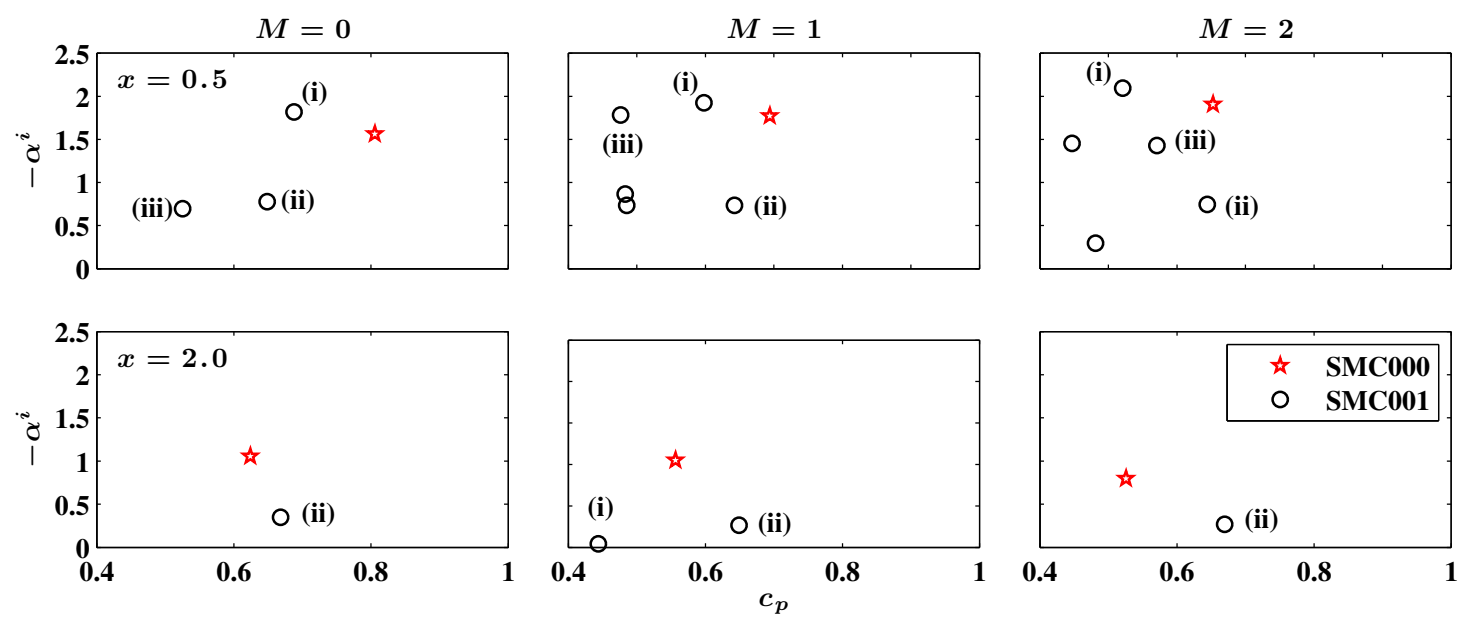

(a)
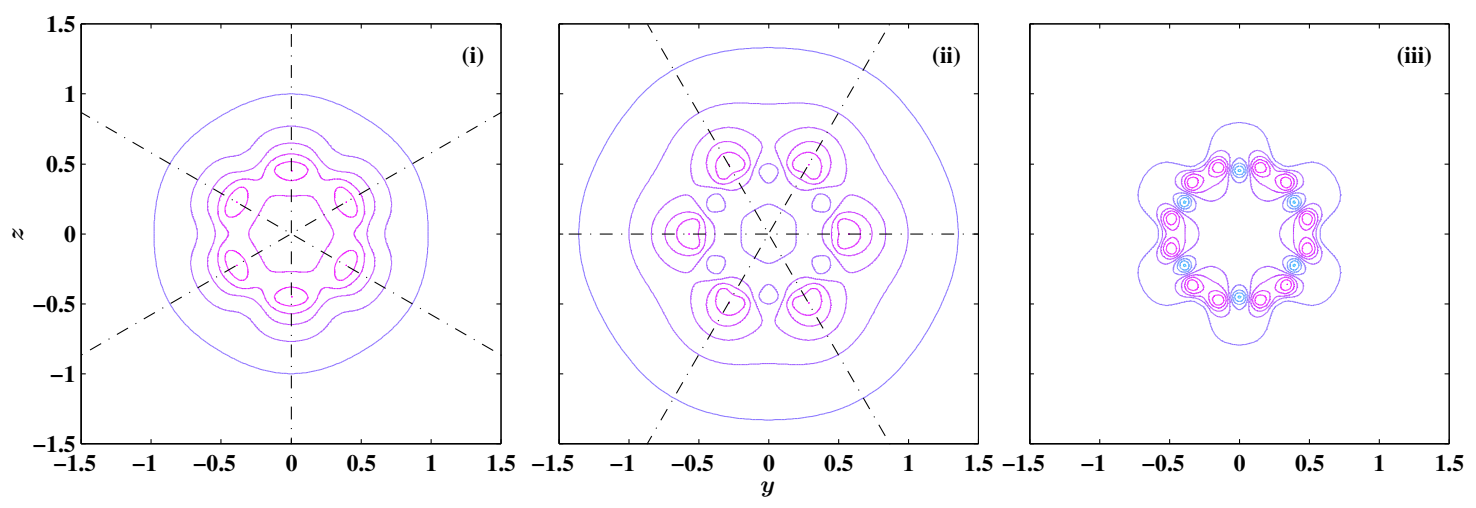

(b)

Figure 4. (a) Eigenspectra (growth rates $-\alpha^{i}$ vs. phase speeds $c_{p}=\omega / \alpha^{r}$ ) for the round (SMC000) and chevroned (SMC001) jets in $S t=0.35$ mode. (b) Real parts of pressure eigenfunctions computed at $x=0.5$ in $\boldsymbol{S t}=0.35, M=0$ mode for the serrated jet. The modes (i)-(iii) correspond to the numbered eigenvalues in fig. 4(a). The violet lines are positive contours; blue lines are negative ones. Contour levels are equally spaced between \pm 0.95 of the maximum absolute values of the respective eigenfunctions. Azimuths of maxima are overlaid for reference.

(between the lobes), whereas mode (ii) peaks at the lobes. The radial gradient is maximum at the flats and minimum at the lobes, which partially explains the relative growth rates of modes (i) and (ii). Mode (iii) has much lower azimuthal coherence, and also decays quickly.

At $x=2.0$, mode (i) is either stable (e.g., in $M=1$ ) or at least less unstable than mode (ii) (e.g., in $M=0$ and 2). This relative ordering of the instability modes is perpetuated further downstream until all normal modes are stable (not shown). This suggests that mode (ii) is more pertinent from a wavepacket perspective since it governs the instability of the shear layer over a much longer axial domain. Mode (i), on the other hand, is a near-nozzle characteristic that is less pertinent for our purposes.

\section{Stability Implications of Mean Flow Variations}

We now report on the modification of the unstable portion of the LST eigen-spectrum as the mean flow field is changed systematically. For this exercise, we focus on the $S t=0.35, M=0$ mode. There are four aspects of interest that we vary separately, with all other aspects maintained at their baseline values; the results are shown in fig. 5 .

Considering the mode (ii) instability (that has been argued to be most important for wavepacket evolution), the growth rate is suppressed as the (a) lobe protrusion is increased, (b) lobe width is decreased, 


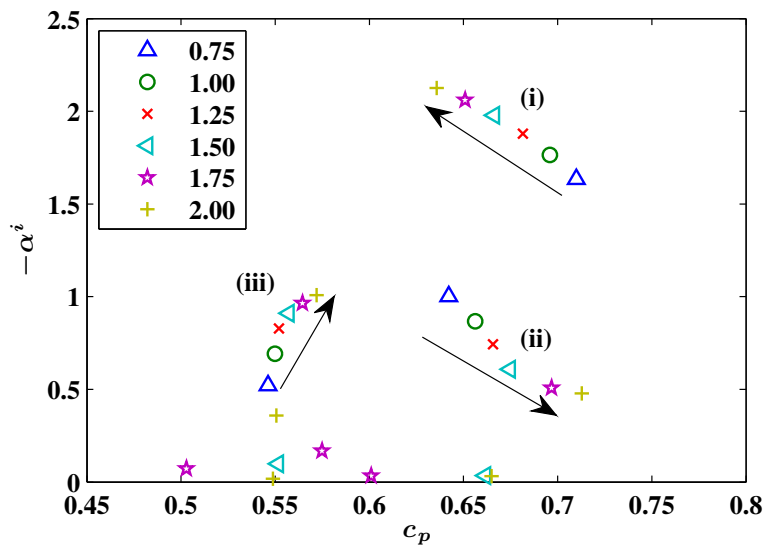

(a)

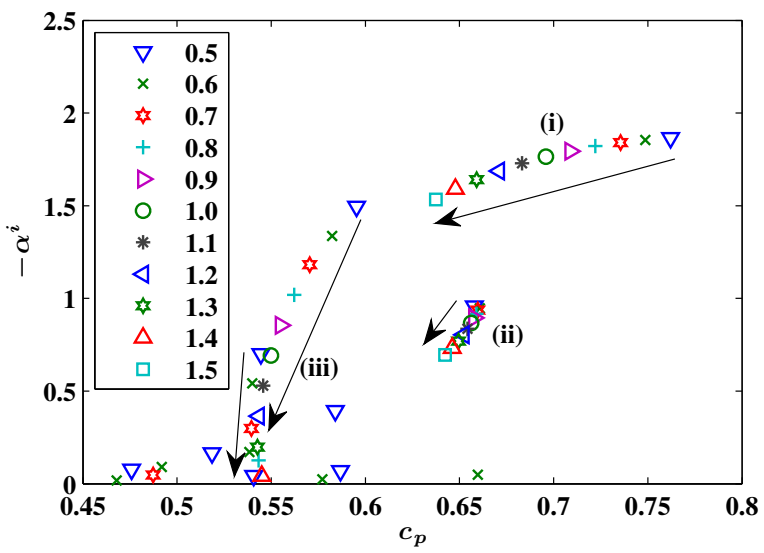

(c)

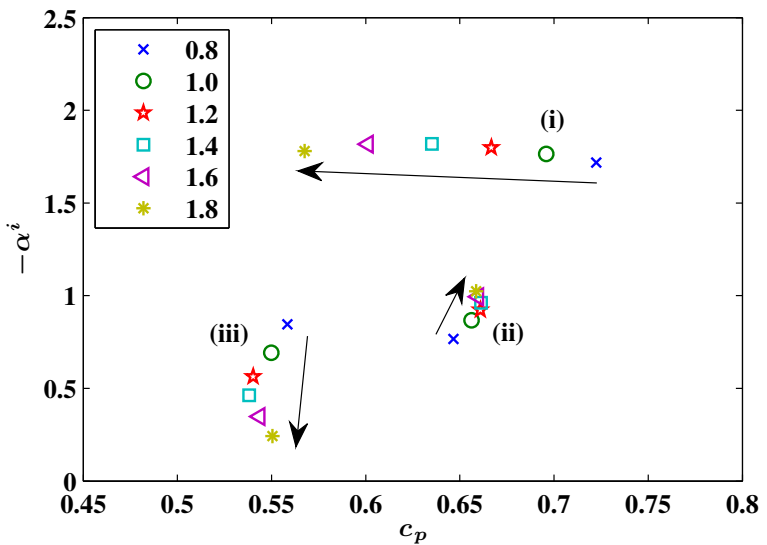

(b)

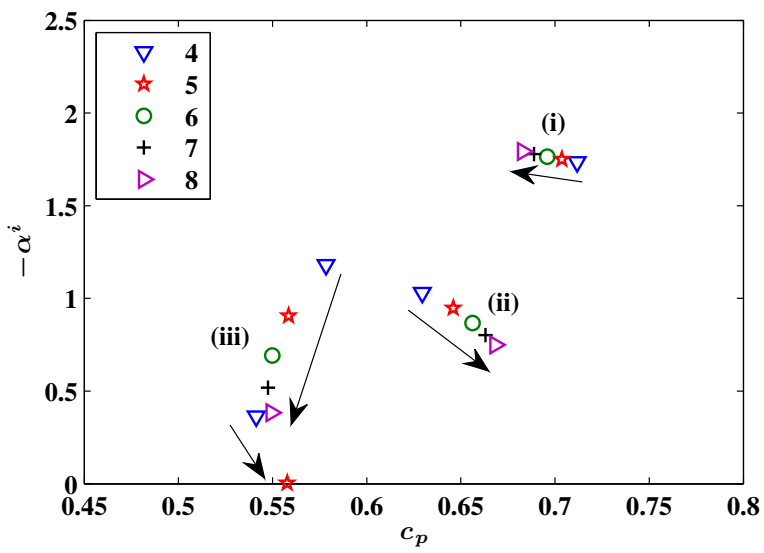

(d)

Figure 5. Eigenspectra of the $S t=0.35, M=0$ mode with individual variations of (a) lobe protrusion, (b) lobe width, (c) average shear layer thickness, and (d) number of lobes.

(c) shear layer thickness is increased, and $(d)$ the number of lobes is increased. The effect of lobe protrusion appears most pronounced, followed by the number of lobes. On the other hand, the phase speed is decreased as the $(a)$ lobe protrusion is decreased, $(b)$ lobe width is decreased, $(c)$ shear layer thickness is increased, and $(d)$ the number of lobes is decreased. However, the percentage changes in the growth rates are much higher compared to those in the phase speeds.

Recalling that a wavepacket's radiation efficiency is reduced if both its growth rate and phase speed are reduced, the effect of the above mean flow modifications on radiated noise can be conjectured as follows. Protrusion of the lobe, a factor that is directly controlled by the degree of impingement of the chevrons in the jet shear layer, strongly reduces growth rate and moderately enhances phase speed of the instability wave. Hence, an optimal protrusion may be hypothesized to exist for minimal jet noise. The same can be said for the number of lobes. The average shear layer thickness should be maximal (obtained by better mixing of the jet shear layer) for noise reduction, as it decreases both the growth rate and phase speed. The width of the lobes of the mean flow profile (relative to the overall circumference) doesn't appear to be a major factor in the instability wave characteristics.

The behaviour of mode (i), the most unstable mode for the baseline mean flow field, is quite different. In fact, the modifications with lobe protrusion and number of lobes are exactly opposite vis-à-vis growth rate as well as phase speed. The variation with average shear layer thickness has the same trend for both modes (i) and (ii). Regarding the width of the lobes, the growth rate is only mildly affected but the phase speed is reduced significantly. Recalling that mode (i) becomes stable further downstream, these effects may not have much impact on the noise characteristics. 

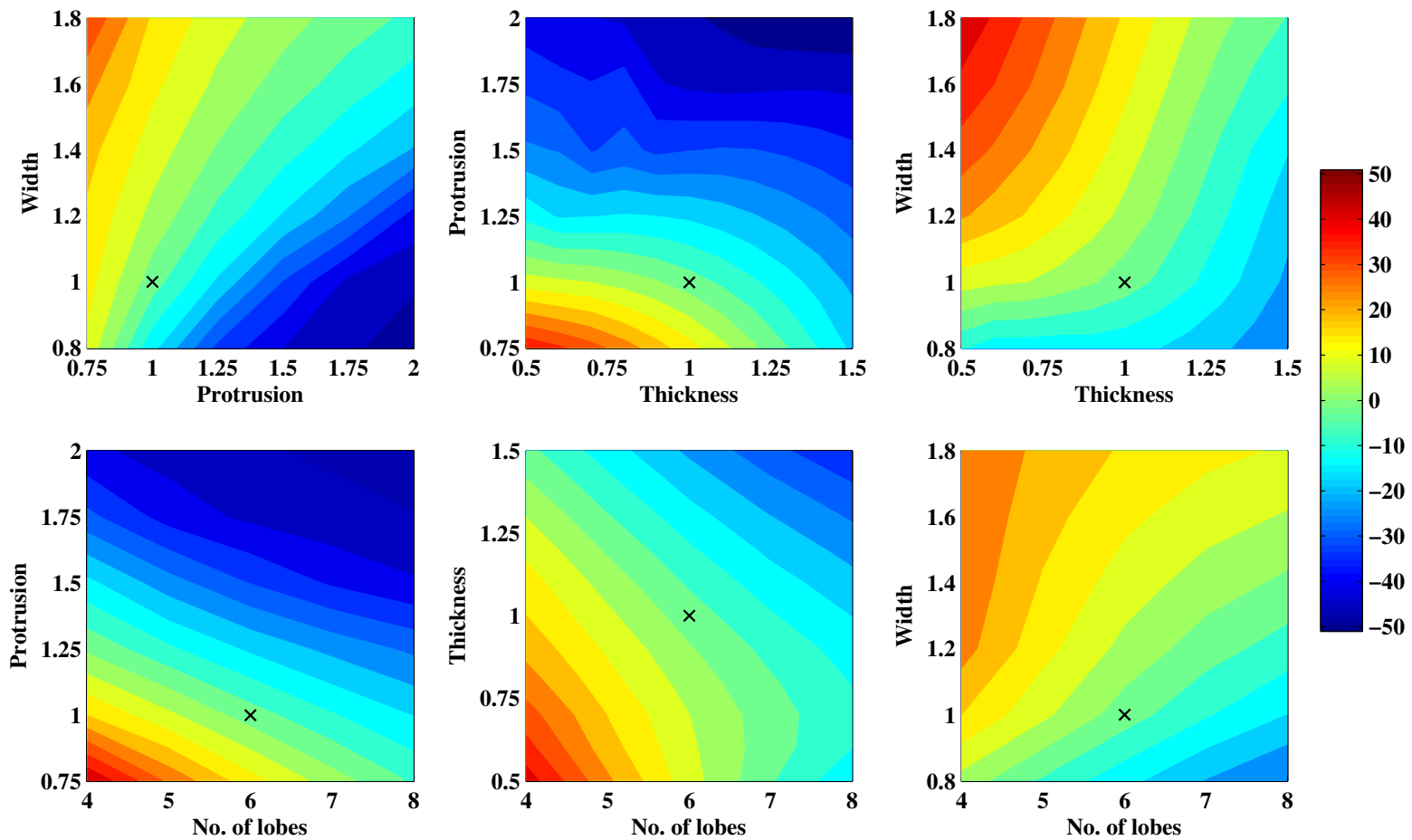

(a)
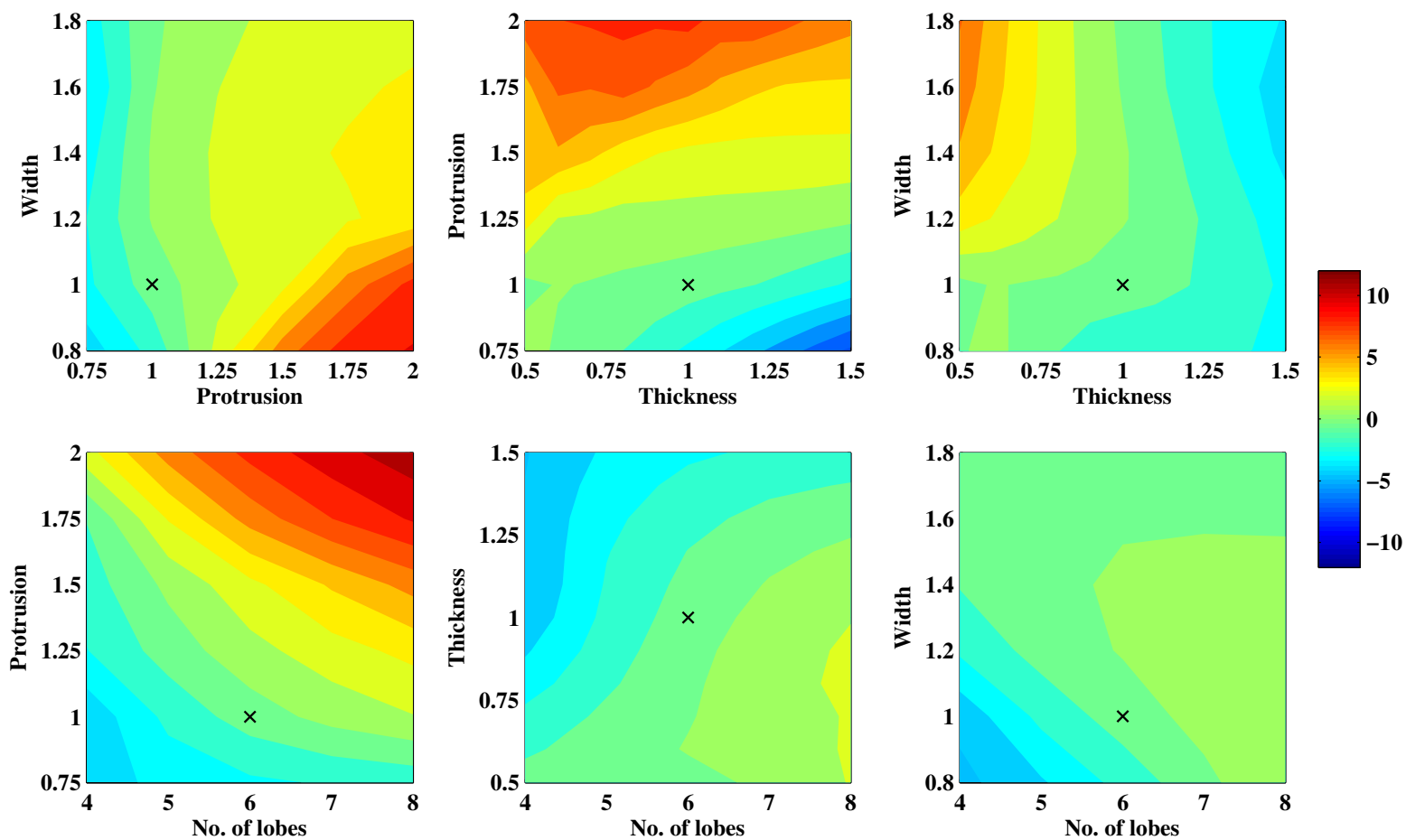

(b)

Figure 6. (a) Growth rates $\left(-\alpha^{i}\right)$ and (b) phase speeds $\left(c_{p}=\omega / \alpha^{r}\right)$ of the mode (ii) instability in $S t=0.35$, $M=0$ with various modifications of the baseline mean flow field. Both quantities are expressed as a percentage of their values in the baseline case, the latter being also indicated by crosses. All modifications are reported as fractions of the baseline values, except for the number of lobes. In each plot, only the aspects shown on the axes are modified, the remaining aspects being held at their baseline values. 
For certain modified mean flows, additional unstable modes appear, apart from the three that exist in the baseline case (see the first sub-plot of fig. 4(a)). This effect is most prominent for very thin shear layers fig. 5(c), but is also seen with higher lobe protrusion in fig. 5(a). The additional mode is typically less unstable than the other ones that already exist in the baseline profile.

The previous discussion has been limited to considerations of each of the four aspects of the mean flow separately. Figure 6 presents the variation of the growth rate and phase speed of the mode (ii) instability as the four aspects of the mean flow are varied in a pairwise manner, holding the other two aspects at their baseline levels. Although no new trend is observed in these contour plots, we confirm the results from fig. 5 . Deeper protrusion of the lobe in conjunction with more number of lobes results in strong suppression of the growth rate, but these factors also moderately increase the phase speed.

\section{Conclusion}

The linear stability characteristics of low-frequency low-azimuthal mode disturbances of the mean flow field in turbulent high speed jets are pertinent for the mixing noise radiated to the aft angle. Chevrons on nozzles create serrations or lobes in the mean flow field that change these stability characteristics, and this effect is a possible explanation for the low-frequency noise reduction observed in such jets. In this work, we identify four aspects of the serrated mean flow as most relevant to the parallel-flow linear stability problem: the number of lobes (corresponding to the number of chevrons), their protrusion and width, and the average shear layer thickness. These aspects are systematically varied starting from a baseline field.

The baseline mean flow profile is chosen as the near-nozzle (i.e., $x=0.5$ ) PIV data of the SMC001 nozzle run at Mach 0.9 cold condition. This serrated profile supports multiple instabilities, the most unstable of which (labelled mode (i) here) actually becomes stable by $x=2$. On the other hand, a less unstable eigenmode (labelled mode (ii)) remains unstable further downstream and thereby governs the overall evolution of the wavepackets in the jet. Thus, we track the mode (ii) instability with modifications of the mean flow profile to understand the possible effect of nozzle serrations on wavepacket dynamics (and hence noise).

We find that increasing the protrusion of the lobes and their overall number serve to greatly decrease the growth rate of the eigenmode (ii) while moderately increasing the phase speed of the same. The average thickness of the shear layer correlates negatively with the instablity. The width of the lobes (as a fraction of the circumference) appears to be mild parameter in the stability problem.

The results found here are corroborated by earlier reports. This is especially true of the decrease in growth rate (and low frequency noise radiation) found with increasing lobe protrusion. The SMC006 nozzle had deeper penetration of the chevron compared to the SMC001 nozzle, and was found to result in lower growth rate of instability ${ }^{12}$ as well as reduced noise radiation at low frequencies. ${ }^{1}$ The SMC004 nozzle had fewer (four in number) chevrons but a similar penetration as the six-chevron SMC001, and was found to have slightly higher noise radiation at low frequencies. ${ }^{1}$

Increasing the chevron impingement not only increases the lobe protrusion near the nozzle but also results in earlier thickening of the shear layer due to increased mixing. ${ }^{26}$ Both these factors decrease the growth rate of the instability. Thus, in the future, the stability effect of the mean flow field will be studied over the entire jet plume. That effort will require mean flow calculations (possibly RANS) to link the chevron geometry to the downstream evolution of the mean flow profile. The latter will also help determine the proper scaling laws for the various parameters, i.e., the behavior at different nozzle exit diameter and initial boundary layer profile.

This study represents a preliminary step towards the creation of a framework for the design of chevron nozzles for aeroacoustic benefit. Other active control devices, like fluidic injectors, zero-net-mass-flux actuators, plasma actuators, etc., also create similar serrations in the mean flow field. Thus, their design/operation may also benefit from this framework.

\section{Acknowledgments}

AS acknowledges support from Industrial Research and Consultancy Center of Indian Institute of Technology Bombay, via the seed grant program. The authors benefited from interactions with André Cavalieri, Francisco Lajús Jr. and Peter Jordan. 


\section{References}

${ }^{1}$ Bridges, J. E. and Brown, C. A., "Parametric testing of chevrons on single flow hot jets," 10th AIAA/CEAS Aeroacoustics Conference, AIAA Paper 2004-2824, 2004.

${ }^{2}$ Alkislar, M. B., Krothapalli, A., and Butler, G. W., "The effect of streamwise vortices on the aeroacoustics of a Mach 0.9 jet," Journal of Fluid Mechanics, Vol. 578, 2007, pp. 139-169.

${ }^{3}$ Jordan, P. and Colonius, T., "Wave Packets and Turbulent Jet Noise," Annu. Rev. Fluid Mech., Vol. 45, 2013, pp. 173195.

${ }^{4}$ Crighton, D. G. and Gaster, M., "Stability of slowly diverging jet flow," Journal of Fluid Mechanics, Vol. 77, No. 2, 1976, pp. 397-413.

${ }^{5}$ Mankbadi, R. and Liu, J. T. C., "Sound Generated Aerodynamically Revisited: Large-Scale Structures in a Turbulent Jet as a Source of Sound," Proc. R. Soc. Lond. A, Vol. 311, No. 1516, 1984, pp. 183-217.

${ }^{6}$ Tam, C. K. W. and Burton, D. E., "Sound generated by instability waves of supersonic flows. Part 1. Two-dimensional mixing layers," Journal of Fluid Mechanics, Vol. 138, 1984, pp. 249-271.

${ }^{7}$ Goldstein, M. E. and Leib, S. J., "The role of instability waves in predicting jet noise," Journal of Fluid Mechanics, Vol. 525, 2005, pp. 37-72.

${ }^{8}$ Suzuki, T. and Colonius, T., "Instability waves in a subsonic round jet detected using a near-field phased microphone array," Journal of Fluid Mechanics, Vol. 565, 2006, pp. 197-226.

${ }^{9}$ Gudmundsson, K. and Colonius, T., "Instability wave models for the near-field fluctuations of turbulent jets," Journal of Fluid Mechanics, Vol. 689, 2011, pp. 97-128.

${ }^{10}$ Cavalieri, A. V. G., Rodríguez, D., Jordan, P., Colonius, T., and Gervais, Y., "Wavepackets in the velocity field of turbulent jets," Journal of Fluid Mechanics, Vol. 730, 2013, pp. 559-592.

${ }^{11}$ Sinha, A., Rodríguez, D., Brès, G., and Colonius, T., "Wavepacket models for supersonic jet noise," Journal of Fluid Mechanics, Vol. 742, 2014, pp. 71-95.

${ }^{12}$ Gudmundsson, K. and Colonius, T., "Spatial stability analysis of chevron jet profiles," 13th AIAA/CEAS Aeroacoustics Conference, AIAA Paper 2007-3599, 2007.

${ }^{13}$ Brown, C. A. and Bridges, J. E., "An analysis of model scale data transformation to full scale flight using chevron nozzles," Tech. rep., NASA TM-2003-212732, 2003.

${ }^{14}$ Bottaro, A., Corbett, P., and Luchini, P., "The effect of base flow variation on flow stability," Journal of Fluid Mechanics, Vol. 476, 2003, pp. 293-302.

${ }^{15}$ Biau, D. and Bottaro, A., "Transient growth and minimal defects: Two possible initial paths of transition to turbulence in plane shear flows," Physics of Fluids, Vol. 16, No. 10, 2004, pp. 3515-3529.

${ }^{16}$ Ben-Dov, G. and Cohen, J., "Instability of optimal non-axisymmetric base-flow deviations in pipe Poiseuille flow," Journal of Fluid Mechanics, Vol. 588, 2007, pp. 189-215.

${ }^{17}$ Lesshafft, L. and Marquet, O., "Optimal velocity and density profiles for the onset of absolute instability in jets," Journal of Fluid Mechanics, Vol. 662, 2010, pp. 398-408.

${ }^{18}$ Cavalieri, A. V. G. and Agarwal, A., "The effect of base-flow changes in Kelvin-Helmholtz instability," 19th AIAA/CEAS Aeroacoustics Conference, AIAA Paper 2013-2088, 2013.

${ }^{19}$ Huerre, P. and Monkewitz, P. A., "Local and global instabilities in spatially developing flows," Annu. Rev. Fluid Mech., Vol. 22, 1990, pp. 437-537.

${ }^{20}$ Khorrami, M. R. and Malik, M. R., "Efficient computation of spatial eigenvalues for hydrodynamic stability analysis," Journal of Computational Physics, Vol. 104, No. 1, 1993, pp. 267-272.

${ }^{21}$ Li, F. and Malik, M. R., "Spectral analysis of parabolized stability equations," Computers \& Fluids, Vol. 26, No. 3, 1997, pp. 279-297.

${ }^{22}$ Lin, C. C., The theory of hydrodynamic stability, Cambridge Univ Press, 1955.

${ }^{23}$ Thompson, K. W., "Time Dependent Boundary Conditions for Hyperbolic Systems," Journal of Computational Physics, Vol. 68, 1987, pp. 1-24.

${ }^{24}$ Mohseni, K. and Colonius, T., "Numerical Treatment of Polar Coordinate Singularities," Journal of Computational Physics, Vol. 157, 2000, pp. 787-795.

${ }^{25}$ Freund, J. B., Compressibility effects in a turbulent axisymmetric mixing layer, Ph.D. thesis, Stanford University, 1997.

${ }^{26}$ Opalski, A. B., Wernet, M. P., and Bridges, J. E., "Chevron nozzle performance characterization using stereoscopic DPIV," 43rd AIAA Aerospace Sciences Meeting and Exhibit, AIAA Paper 2005-444, 2005. 